\title{
Safety and patient-reported outcomes in index ablation versus repeat ablation in atrial fibrillation: insights from the German Ablation Registry
}

\author{
Shinwan Kany ${ }^{1}$. Johannes Brachmann ${ }^{2}$. Thorsten Lewalter ${ }^{3} \cdot$ Karl-Heinz Kuck $^{4}$. Dietrich Andresen ${ }^{5}$. \\ Stephan Willems ${ }^{6} \cdot$ Ellen Hoffmann $^{7} \cdot$ Lars Eckardt $^{8} \cdot$ Dierk Thomas $^{9} \cdot$ Matthias Hochadel $^{10} \cdot$ Jochen Senges $^{10}$. \\ Andreas Metzner ${ }^{1} \cdot$ Andreas Rillig $^{1}$
}

Received: 20 July 2020 / Accepted: 12 October 2020 / Published online: 28 October 2020

(c) The Author(s) 2020

\begin{abstract}
Background Pulmonary vein isolation is an established strategy for catheter ablation of atrial fibrillation (AF). However, in a significant number of patients, a repeat procedure is mandatory due to arrhythmia recurrence. In this study, we report safety data and procedural details of patients undergoing index ablation versus repeat ablation in a registry-based real-life setting. Methods Patients from the German Ablation Registry, a prospective, multicentre registry of patients undergoing ablation between January 2007 and January 2010 were included.

Results A total of 4155 patients were enrolled in the study. Group I (index ablation) consisted of 3377/4155 (82.1\%) and group II (repeat ablation) of 738/4155 (17.9\%). Patients in group I had a significantly higher ratio of paroxysmal AF (69.3\% vs $61.9 \%, p<0.001)$ and significantly less persistent $\mathrm{AF}(30.7 \%$ vs $38.1 \%, p<0.001)$. The repeat group showed significantly lower mean RF application duration (2580 s. vs 1960, $p<0.001$ ), less fluoroscopy time (29 min. vs. 27 min., $p<0.001$ ), less mean dose area product (DAP) ( $3744 \mathrm{cGy} \times \mathrm{cm}^{2}$ vs $\left.3325 \mathrm{cGy} \times \mathrm{cm}^{2}, p=0.001\right)$, and shorter study duration (181.2 min. vs 163.6 min., $p<0.001)$. No statistical difference between the groups was found in terms of mortality $(0.3 \% \mathrm{vs} 0.1 \%, p=0.39)$, MACE $(0.4 \%$ vs $0.3 \%, p=0.58)$, MACCE $(0.8 \%$ vs $0.6 \%, p=0.47)$, composite safety endpoint $(1.5 \%$ vs $1.4 \%, p=0.76)$, and arrhythmia recurrence $(43.8 \%$ vs $41.9 \%, p=0.37)$ during 1 -year follow-up. Both groups reported to have improved or no symptoms $(80.4 \%$ vs $77.8 \%, p=0.13)$.

Conclusion Repeat catheter ablation is safe and provides a symptomatic relief comparable to index ablation. Repeat procedures are significantly shorter and use less fluoroscopy.
\end{abstract}

Keywords Atrial fibrillation $\cdot$ Repeat ablation $\cdot$ Patient-reported outcomes

Andreas Rillig

a.rillig@uke.de

1 Department of Cardiology, University Heart and Vascular Center Hamburg-Eppendorf, Martinistraße 52,

20251 Hamburg, Germany

2 Department of Cardiology, Angiology and Pneumology, Coburg Hospital, Coburg, Germany

3 Department of Medicine-Cardiology and Intensive Care, Hospital Munich-Thalkirchen, Munich, Germany

4 LANS Cardiology, Hamburg, Germany

5 Department of Cardiology, Evangelisches Krankenhaus Hubertus, Berlin, Germany
6 Department of Cardiology, Asklepios Klinik St. Georg, Hamburg, Germany

7 Dept. of Cardiology and Internal Intensive Care Medicine, Heart Center Munich-Bogenhausen, Munich Clinic Bogenhausen, Munich, Germany

8 Department of Cardiology (Electrophysiology), University Hospital Muenster, Muenster, Germany

9 Department of Cardiology, University of Heidelberg, Heidelberg, Germany

10 Stiftung Für Herzinfarktforschung (IHF), Ludwigshafen, Germany 


\section{Introduction}

Atrial fibrillation (AF) is the most common arrhythmia affecting humans and is, in addition to oftentimes typical symptoms and reduced quality of life, associated with a rise of associated mortality [1]. According to the most recent guidelines, catheter ablation via pulmonary vein isolation (PVI) is a class I recommendation for patients with either paroxysmal or persistent atrial fibrillation after failed antiarrhythmic drug therapy [2]. Recently, randomized-controlled trials comparing radiofrequency (RF) and cryoballoon (CB) ablation in AF showed that either method is associated with high freedom of arrhythmias [3-5]. A significant portion of patients is in need of a repeat ablation due to re-connection of previously isolated pulmonary veins [4]. Currently, more progressive forms like persistent $\mathrm{AF}$ and long-standing persistent $\mathrm{AF}$ show a greater tendency to require more than one ablation to increase the probability of long-lasting success [4]. No ablation strategy besides PVI has been proven to comprehensively and reproducibly reduce the recurrence of AF after index ablation. Additionally, the 2020 ESC guidelines recommend collecting patient-reported outcomes (PRO) to assess success of patients care [2]. Furthermore, repeated catheter ablation is given a class IIa recommendation if the index procedure provided symptomatic relief [2].

Large real-world data comparing repeat vs. index ablation and differential ablation strategies remain scarce. Additionally, the patient cohort undergoing repeat ablation as well as procedural safety and efficacy have only been characterized in select populations.

This study aims to analyze repeat procedures compared to index procedures regarding procedural details as well as safety and PRO such as symptomatic burden and satisfaction in a prospective, multicentre registry.

\section{Methods}

\section{German ablation registry structure}

The non-profit organization "Institut für Herzinfarktforschung" (IHF, Ludwigshafen, Germany) is supervising the prospective, multi-center German Ablation Registry. Out of 55 participating German centers, 41 provided cases with AF catheter ablation.

\section{Patient cohort}

Patients with age $>18$ years undergoing catheter ablation for symptomatic AF between January 2007 and January 2010 at participating centers were enrolled. Patients with AV node ablation or with long-standing persistent AF were excluded from the analysis. The cohort was divided into two groups undergoing either index (group I) or repeat ablation (group II) and compared. Written consent for catheter ablation and participation for the registry were obtained beforehand. The ethics committee of the Rhineland-Palatinate State Medical Council (Landesärztekammer Rheinland-Pfalz) approved the study (No. 837.026.07 (5561)).

\section{Procedural methods for catheter ablation}

Procedures were performed in accordance with standard protocols at participating centers. Procedure protocols have been described in detail before [6-8]. Transthoracic echocardiography was performed to assess left-ventricular ejection fraction (LVEF) and left atrial (LA) diameter. To rule out intracardiac thrombi, transesophageal echocardiography was performed. Additional pre-procedural imaging was left to each participating partner. Procedures were conducted under deep sedation. Hemodynamic parameters including, but not limited to, blood pressure, heart rate, and oxygen saturation were monitored.

An activated clotting time (ACT) target of 300-400 s was maintained throughout the procedure. Ablation and transseptal access were guided by fluoroscopy during CB and RF. Only the first-generation CB (Medtronic, Minneapolis, MN, USA) was available for the procedures. In the latter case, electroanatomical mapping (EAM) systems such as CARTO or NavX were used according to the operator's preference. PVI was confirmed using diagnostic catheters. Additional ablation strategies may include linear lesions of either right atrium (RA) or LA such as the cavo-tricuspid isthmus (CTI) area, as well as ablation of complex fractionated atrial electrograms (CFAEs), and were left to the operator's choice. During CB ablation, continuous monitoring of the phrenic nerve was achieved via fluoroscopy or pacing maneuvers. After the procedure, pressure bandages or figure of eight sutures were applied to the groin area. Anti-arrhythmic drug therapy (AAD) and post-procedural anticoagulation management were left to standard procedures at participating sites.

\section{Registry management and clinical follow-up}

The IHF is managing project development, data acquisition, and clinical monitoring. Physicians and study nurses at participating sites entered data for baseline characteristics, procedural details, and 30-day follow-up. Any arrhythmia was documented via ECG tracings and/or Holter recording. The IHF conducted a 1-year follow-up using electronic health records and telephone calls for standardized questionnaires. In between this time-period, clinical follow-up occurred at each participating center's discretion including (but not mandatory) Holter monitoring. For assessment of 
AF symptomatic burden, the severity of AF scale (SAF) was used. Electronic data management with Internet-based care report forms (EBogen $\odot$, developed by the IHF) using secure encryption was employed. Statistical analysis and biomedical models were carried out by the IHF.

\section{Outcomes}

The primary outcome was the acute procedural success and safety. Major adverse cardiac event (MACE) was defined as death or myocardial infarction (MI). Major adverse cardiac and cerebrovascular events (MACCE) was defined as death, MI, or stroke. Composite safety endpoint was defined as death, MI, stroke, or major bleeding.

Secondary endpoints were patient survival, long-term procedural success, and safety. Additionally, patient-oriented outcome such as quality of life and satisfaction with treatment were considered secondary endpoints.

Potential periprocedural complications were categorized into severe, moderate, and minor complications.

\section{Statistical analysis}

Normally distributed continuous data are shown as means \pm standard deviation (SD), otherwise given as medians with first and third quartiles. Categorical data are shown in relative percentages and absolute values. Statistical differences between both groups were compared using either Mann-Whitney-Wilcoxon test or with a Chi-square test. For rates of in-hospital complications, Fisher's exact test was used. The 12-month event-rates of MACCE (composite outcome of death, myocardial infarction, and stroke), MACE (composite outcome of death and myocardial infarction), and composite safety endpoint (composite endpoint of death, myocardial infarction, stroke, and major bleeding) were calculated by the Kaplan-Meier method. The aforementioned outcomes were compared between age groups using the log-rank test. All statistical comparisons were two-sided, and $P$ values $<0.05$ were considered statistically significant. Analyses were performed using the Statistical Analysis System (SAS, Version 9.4, SAS Institute Inc., Cary, NC, USA).

\section{Results}

\section{Patient cohort and baseline parameters}

A total of 4155 patients were included in the study. Group I (index ablation) consisted of 3377/4155 (82.1\%) and group II (repeat ablation) of 738/4155 (17.9\%). The proportion of female patients was higher in patients of the index cohort (33.5\% vs $29.1 \%, p=0.021)$, whereas median age was lower (62 vs 63 years; $p=0.021$ ). Patients from group I compared to group II showed no statistically different comorbidities in diabetes mellitus (DM), chronic kidney disease (CKD), hypertension, and prior stroke as well as chronic obstructive pulmonary disease (COPD). Both groups had comparable proportions of prior device implantation like pacemaker (PM), implantable cardioverter defibrillator (ICD), or cardiac resynchronisation therapy (CRT). Group I and group II were similar with regards to comorbidities such as coronary artery disease (CAD), prior myocardial infarction, and cardiomyopathy. Patients undergoing index ablation were less likely to have valvular heart disease $(6.6 \%$ vs $9.1 \%$, $p=0.016)$. LVEF was preserved in either group at $86.8 \%$ vs $88.0 \%(p=0.40)$. Group I had a significantly lower portion of patients with severe symptoms (New York Heart Association class, NYHA $2+$ ) compared to group II (39.7\% vs $49.4 \%, p=0.004)$. $\mathrm{CHA}_{2} \mathrm{DS}_{2}$-VASc Scores were comparable in both groups $(1.8 \pm 1.3$ vs $1.7 \pm 1.2, p=0.63)$. Patients at index ablation (group I) had a significantly higher proportion of paroxysmal AF $(69.3 \%$ vs $61.9 \%, p<0.001)$ and significantly less persistent $\mathrm{AF}(30.7 \%$ vs $38.1 \%, p<0.001)$. Patients with long-standing persistent AF were not included in the analysis. An overview of the traits of each group is given in Table 1.

\section{Procedural details}

Before ablation rhythm at ablation in both groups was comparable at sinus rhythm (SR) and AF. Compared to repeat ablation, PVI at index procedure was carried out significantly more often using a circumferential approach $(87.9 \%$ vs $78.0 \%, p<0.001)$ and less-segmental isolation $(12.4 \%$ vs $16.0 \%, p=0.008)$. Linear lesions were significantly less common in the index group compared to the repeat group (14.6\% vs $26.6 \%, p<0.001)$. Of those lesions, the index group demonstrated a lower proportion of linear lesions in the LA ( $49.2 \%$ vs $60.7 \%, p=0.006)$, but no difference in RA lesions (56.7\% vs $57.7 \%, p=0.82)$ and CTI ablation $(97.1 \%$ vs $92.9 \%, p=0.055)$. CFAE ablation was conducted significantly less often in the index ablation group ( $7.8 \%$ vs $25.1 \%, p<0.001$ ). Both conventional mapping as well as EAM were utilized similarly between the groups. Pre-procedural imaging was statistically more prevalent at index ablation $(25.2 \%$ vs $16.7 \%, p<0.001)$, driven by computed tomography (CT) $(18.9 \%$ vs $12.3 \%, p<0.001)$. Group I was treated with significantly less RF energy $(77 \%$ vs $94.6 \%, p<0.001)$ and more CB ablation $(21.7 \%$ vs $5.4 \%$, $p<0.001$ ). Acute procedural success was the same for both groups $(96.3 \%$ vs $97.0 \%, p=0.36)$. The index group showed significantly higher mean RF application duration (2580 s. vs $1960, p<0.001$ ), longer fluoroscopy time (29 $\mathrm{min}$. 27 min., $p<0.001$ ), more mean dose area product (DAP) (3744 cGy $\times \mathrm{cm}^{2}$ vs $\left.3325 \mathrm{cGy} \times \mathrm{cm}^{2}, p=0.001\right)$, and longer 
Table 1 Baseline characteristics of both patients undergoing index versus repeat ablation

\begin{tabular}{llll}
\hline & Index $(n=3377)$ & Repeat $(n=738)$ & $\begin{array}{l}\text { Odds ratio (95\%-CI) } \\
\text { or p-value }\end{array}$ \\
\hline Age (years) & $62(54 ; 68)$ & $63(55 ; 68)$ & $P=0.69$ \\
Female sex & $33.5 \%$ & $29.1 \%$ & $1.23(1.03-1.46)$ \\
Diabetes mellitus & $7.8 \%$ & $6.8 \%$ & $1.17(0.86-1.60)$ \\
Chronic kidney disease & $2.1 \%$ & $2.8 \%$ & $0.76(0.21-2.72)$ \\
Hypertension & $61.1 \%$ & $64.5 \%$ & $0.86(0.56-1.32)$ \\
Stroke & $5.6 \%$ & $4.6 \%$ & $1.24(0.47-3.26)$ \\
COPD & $1.2 \%$ & $2.8 \%$ & $0.44(0.11-1.73)$ \\
Cardiac device (PM, ICD, CRT) & $5.9 \%$ & $6.2 \%$ & $0.95(0.68-1.32)$ \\
Pacemaker & $4.4 \%$ & $5.3 \%$ & $1.20(0.84-1.72)$ \\
ICD & $1.4 \%$ & $0.9 \%$ & $0.66(0.30-1.47)$ \\
CRT & $0.1 \%$ & $0.1 \%$ & $2.29(0.21-25.28)$ \\
Coronary artery disease & $18.2 \%$ & $16.1 \%$ & $1.15(0.93-1.43)$ \\
Prior myocardial infarction & $4.6 \%$ & $6.2 \%$ & $0.73(0.52-1.03)$ \\
Cardiomyopathy (HCM, DCM) & $3.9 \%$ & $3.8 \%$ & $1.04(0.69-1.58)$ \\
Valvular heart disease & $6.6 \%$ & $9.1 \%$ & $0.70(0.53-0.94)$ \\
LVEF preserved (> 50\%) & $86.8 \%$ & $88.0 \%$ & $0.90(0.69-1.17)$ \\
CHA ${ }_{2}$ DS ${ }_{2}$-VASc Score & $1.8 \pm 1.3$ & $1.7 \pm 1.2$ & $P=0.63$ \\
NYHA 2+ & $39.7 \%$ & $49.4 \%$ & $0.67(0.51-0.88)$ \\
Atrial fibrillation type & & & $P<0.001$ \\
Paroxysmal & $69.3 \%$ & $61.9 \%$ & $P<0.001$ \\
Persistent & $30.7 \%$ & $38.1 \%$ & \\
\hline
\end{tabular}

All values given as percentages or mean with standard deviation or quartiles. $P$ value $<0.05$ was considered significant

$C I$ confidence interval, $C O P D$ Chronic Obstructive Pulmonary Disease; $P M$ Pacemaker; $I C D$ Implantable Cardioverter Defibrillator; CRT Cardiac Resynchronization Therapy; HCM Hypertrophic Cardiomyopathy; DCM Dilatative Cardiomyopathy; LVEF Left-Ventricular Ejection Fraction; NYHA New York Heart Association study duration (181.2 $\mathrm{min}$. vs $163.6 \mathrm{~min} ., p<0.001)$. An overview of procedural data is given in Table 2 .

\section{In-hospital complications and safety}

There was one death in group I during hospital stay. Severe non-fatal adverse events (AE) and moderate non-fatal AE were comparable in both groups. Mild AE were significantly less for the repeat ablation cohort (3.8\% vs $2.0 \%, p=0.024)$. Detailed data are presented in Table 3. Index ablation and repeat ablation performed comparably in MACE $(0.1 \%$ vs $0.0 \%, p=1.0)$, MACCE $(0.3 \%$ vs $0 \%, p=0.23)$ and composite safety endpoint ( $1.2 \%$ vs $0.5 \%, p=0.12)$. In-hospital recurrence of arrhythmia was significantly higher after index ablation compared to repeat procedure $(7.4 \%$ vs. $4.5 \%$, $p=0.004)$.

\section{Long-term follow-up: complication and safety}

Follow-up information was obtained for 3304 patients (97.9\%) in group I after a median of 457 days post-hospital discharge and for 718 patients $(97.3 \%)$ in group II after a median of 463 days. There was no statistical difference between the groups in terms of mortality $(0.3 \%$ vs $0.1 \%$, $p=0.39)$, MACE $(0.4 \%$ vs $0.3 \%, p=0.58)$, MACCE $(0.8 \%$ vs $0.6 \%, p=0.47)$, and composite safety endpoint $(1.5 \%$ vs $1.4 \%, p=0.76)$. Both groups were comparable with regard to severe non-fatal $\mathrm{AE}(1.6 \%$ vs $2.4 \%, p=0.20)$ and moderate non-fatal AE $(8.3 \%$ vs $7.6 \%, p=0.60)$. Incidence of PV stenosis $(0.1 \%$ vs $0.0 \%, p=0.35)$, phrenic nerve injury $(0.5 \%$ vs $0.3 \%, p=0.45)$, and atrio-esophageal fistula ( $0 \%$ vs $0.1 \%, p=0.24)$ were not significantly different. Incidence of new cardiac device implantation was similar in both groups (2.5\% vs $3.0 \%, p=0.45)$. In Fig. 1, a graph with the 366-day safety data is given.

\section{Long-term follow-up: symptoms, recurrence, and satisfaction}

Compared to the index cohort, patients after repeat ablation had comparable symptoms such as dyspnea and less, albeit not significantly, angina ( $16 \%$ vs $11.9 \%, p=0.052)$. Both groups reported to have improved or no symptoms at high ratios $(80.4 \%$ vs $77.8 \%, p=0.13)$. There was no difference in 
Table 2 Procedural details of AF patients undergoing index versus repeat ablation

\begin{tabular}{|c|c|c|c|}
\hline & Index $(n=3377)$ & Repeat $(n=738)$ & $p$ value \\
\hline \multicolumn{4}{|l|}{ Energy form used } \\
\hline Radiofrequency & $77.0 \%$ & $94.6 \%$ & $<0.001$ \\
\hline Cryoballoon & $21.7 \%$ & $5.4 \%$ & $<0.001$ \\
\hline \multicolumn{4}{|l|}{ Rhythm before ablation } \\
\hline Sinus rhythm & $68.5 \%$ & $68.2 \%$ & 0.85 \\
\hline Atrial fibrillation & $31.5 \%$ & $31.8 \%$ & 0.85 \\
\hline \multicolumn{4}{|l|}{ Pulmonary vein isolation } \\
\hline Circumferential & $87.9 \%$ & $78.0 \%$ & $<0.001$ \\
\hline Segmental & $12.4 \%$ & $16.0 \%$ & 0.008 \\
\hline \multicolumn{4}{|l|}{ Ablation strategy } \\
\hline Linear lesion & $14.6 \%$ & $26.6 \%$ & $<0.001$ \\
\hline Location LA & $49.2 \%$ & $60.7 \%$ & 0.006 \\
\hline Location RA & $56.7 \%$ & $57.7 \%$ & 0.82 \\
\hline Location CTI & $97.1 \%$ & $92.9 \%$ & 0.055 \\
\hline CFAE & $7.8 \%$ & $25.1 \%$ & $<0.001$ \\
\hline Irrigated-tip catheters & $70.0 \%$ & $90.7 \%$ & $<0.001$ \\
\hline \multicolumn{4}{|l|}{ Mapping, imaging, and study } \\
\hline Conventional mapping & $39.3 \%$ & $40.2 \%$ & 0.63 \\
\hline EAM & $58.0 \%$ & $58.3 \%$ & 0.88 \\
\hline Pre-procedural imaging & $25.2 \%$ & $16.7 \%$ & $<0.001$ \\
\hline Mean RF application duration (sec) & $2580(1657 ; 3656)$ & $1960(1038 ; 3405)$ & $<0.001$ \\
\hline Mean fluoroscopy time (min) & $29(20 ; 46)$ & $27(17 ; 44)$ & $<0.001$ \\
\hline Mean dose area product $\left(\mathrm{cGY} \times \mathrm{cm}^{2}\right)$ & $3744(1898 ; 7234)$ & $3325(1754 ; 6229)$ & 0.001 \\
\hline Mean study duration (min) & $181.2 \pm 71.9$ & $163.6 \pm 67.1$ & $<0.001$ \\
\hline
\end{tabular}

All values are given as percentages or mean with standard deviation or quartiles. $P$ value $<0.05$ was considered significant

$L A$ Left Atrium; RA Right Atrium; CTI Cavo-tricuspid Isthmus; CFAE Complex Fractioned Atrial Electrograms; EAM Electroanatomical Mapping; RF Radiofrequency; sec Seconds; min Minutes the number of hospitalisations. Patients after repeat ablation were similarly satisfied or partially satisfied with the given treatment compared to the index group. In both groups, the number of unsatisfied patients was statistically similar. In both groups, the feeling of safety during treatment was equally high. Patient-oriented outcomes are visualized in Fig. 2. Arrhythmia recurrence rates were not significantly different in both groups. A graphical comparison to other index vs repeat catheter ablation studies is given in Fig. 3 .

\section{Medical therapy at discharge}

In both groups, antiarrhythmic therapy at discharge was similar in both groups (Table 4). Therapy with class I AAD $(32.6 \%$ vs $30.1 \%, p=0.18)$ and class III AAD $(21.4 \%$ vs $24.4 \%, p=0.081)$ was common. The therapy was mostly unchanged after the procedure, and in only $2.6 \%$ vs $2.8 \%$ $(p=0.9)$ of the cases, class III AAD was added to existing medication. The antithrombotic regime was mostly comprised of vitamin $\mathrm{K}$ antagonists $(89.6 \%$ vs $91 \%, p=0.26$ ) which were mostly bridged by unfractionated heparin $(8.3 \%$ vs $3.9 \%, p<0.001)$ or low-molecular-weight heparin $(63.3 \%$ vs $70.3 \%, p<0.001)$.

\section{Discussion}

In this large, prospective multi-center cohort, we demonstrate that index as well as repeat ablation in AF are safe and provide significant improvement in symptoms and patients' satisfaction. Additionally, these data demonstrated more extensive ablation strategies in repeat ablation with shorter EP study and fluoroscopy time as compared to index ablation.

Despite adequate rate control, symptomatic AF patients are often in need for rhythm control strategies such as PVI to reduce symptoms and improve quality of life. The need for the establishment of patient-oriented outcomes in clinical trials has been reiterated $[9,10]$.

However, early studies comparing rhythm control strategies in AF such as RACE and SAFE-T excluded patients with symptoms akin to NYHA class III or IV $[11,12]$. In 
Table 3 Procedural-related complications during hospital stay
Fig. 1 366-day safety followup by Kaplan-Meier method. MACE: composite endpoint of death and myocardial infarction; MACCE: composite endpoint of death, myocardial infarction, and stroke; Composite Safety Endpoint; composite endpoint of death, myocardial infarction, stroke, and major bleeding. $P$ value $<0.05$ was considered significant and calculated logrank test

\begin{tabular}{|c|c|c|c|}
\hline & Index & Repeat & $p$ value \\
\hline Severe complications & $1.2 \%(41 / 3373)$ & $0.5 \%(4 / 738)$ & 0.12 \\
\hline Myocardial infarction & $0.1 \%(2 / 3373)$ & $0.0 \%(0 / 738)$ & 1.00 \\
\hline Stroke & $0.2 \%(7 / 3373)$ & $0.0 \%(0 / 738)$ & 0.36 \\
\hline Major bleeding requiring intervention & $0.9 \%(32 / 3374)$ & $0.5 \%(4 / 738)$ & 0.38 \\
\hline Moderate complications & $3.2 \%(95 / 2970)$ & $2.2 \%(14 / 640)$ & 0.20 \\
\hline Transient ischemic attack & $0.1 \%(5 / 3373)$ & $0.0 \%(0 / 738)$ & 0.59 \\
\hline Cardiopulmonary resuscitation & $0.0 \%(0 / 3373)$ & $0.1 \%(1 / 737)$ & 0.18 \\
\hline Aneurysmal hematoma. AV fistula & $1.1 \%(38 / 3376)$ & $1.1 \%(8 / 738)$ & 1.00 \\
\hline Infection of puncture site & $0.0 \%(1 / 2969)$ & $0.0 \%(0 / 641)$ & 1.00 \\
\hline Pericardial effusion & $1.1 \%(33 / 2969)$ & $0.5 \%(3 / 641)$ & 0.19 \\
\hline Persistent AV block & $0.0 \%(0 / 2969)$ & $0.2 \%(1 / 641)$ & 0.18 \\
\hline AV block III & $0.1 \%(2 / 2969)$ & $0.0 \%(0 / 641)$ & 1.00 \\
\hline Sepsis & $0.0 \%(0 / 2969)$ & $0.0 \%(0 / 641)$ & \\
\hline Endocarditis & $0.0 \%(0 / 2969)$ & $0.0 \%(0 / 641)$ & \\
\hline Pulmonary embolism & $0.0 \%(1 / 2969)$ & $0.0 \%(0 / 641)$ & 1.00 \\
\hline Pneumothorax & $0.2 \%(7 / 2969)$ & $0.0 \%(0 / 641)$ & 0.62 \\
\hline Hemothorax & $0.1 \%(4 / 2969)$ & $0.0 \%(0 / 641)$ & 1.00 \\
\hline Emergent cardiac surgery & $0.1 \%(3 / 2969)$ & $0.0 \%(0 / 641)$ & 1.00 \\
\hline Phrenic nerve palsy & $0.5 \%(15 / 3013)$ & $0.0 \%(0 / 643)$ & 0.089 \\
\hline Pulmonary vein stenosis & $0.1 \%(2 / 2969)$ & $0.2 \%(1 / 641)$ & 0.44 \\
\hline Atrio-esophageal fistula & $0.0 \%(0 / 2969)$ & $0.0 \%(0 / 641)$ & \\
\hline Minor complications & $3.8 \%(113 / 2978)$ & $2.0 \%(13 / 641)$ & 0.024 \\
\hline Minor bleeding & $3.3 \%(112 / 3373)$ & $1.6 \%(12 / 737)$ & 0.012 \\
\hline New AV block $\mathrm{I}^{\circ}$ or $\mathrm{II}^{\circ}$ & $0.0 \%(1 / 2969)$ & $0.2 \%(1 / 641)$ & 0.32 \\
\hline New Right or left bundle branch block & $0.0 \%(0 / 2969)$ & $0.0 \%(0 / 641)$ & \\
\hline
\end{tabular}

All values given as percentages with total numbers in brackets. $P$ values were calculated by Fisher's exact test and considered significant if $<0.05$. $^{*}$

$2,0 \%$

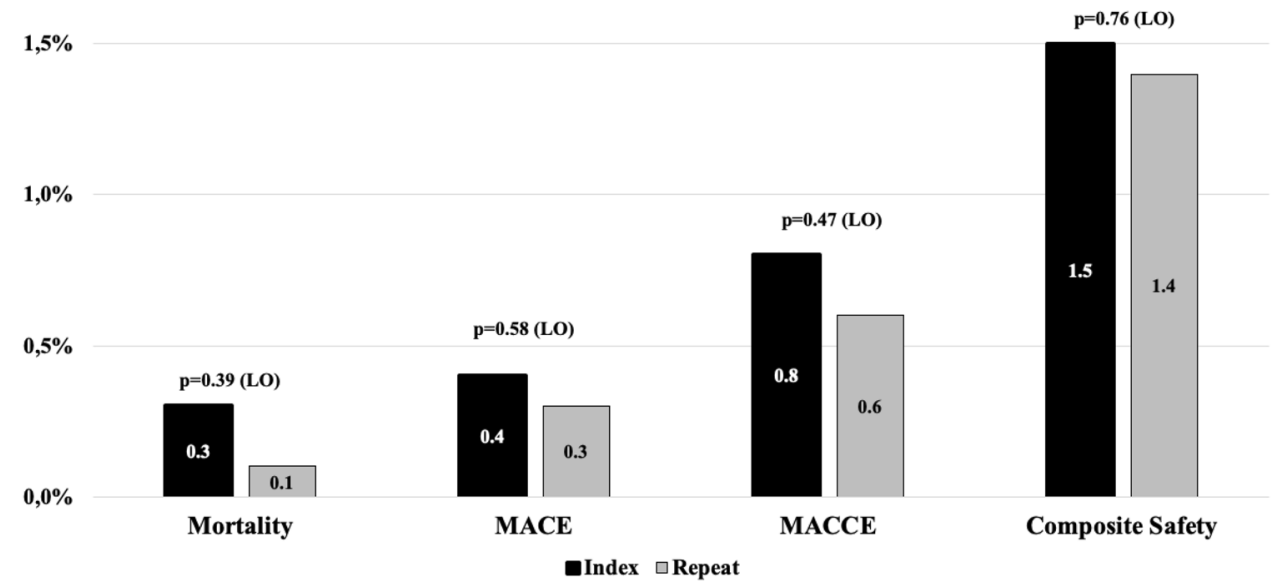

our study, we showed that a significant portion of patients (39.7\%) undergoing index ablation reports symptoms of NYHA II or greater. In the cohort undergoing repeat ablation, almost half $(49.4 \%)$ reported this severe symptomatic burden. Comparatively, in the recent CABANA trial, only $34.5 \%$ (in the ablation group) and 34\% in the medical therapy group reported symptoms of $\geq$ NYHA II [13]. This may suggest that our real-world cohort undergoing AF ablation 


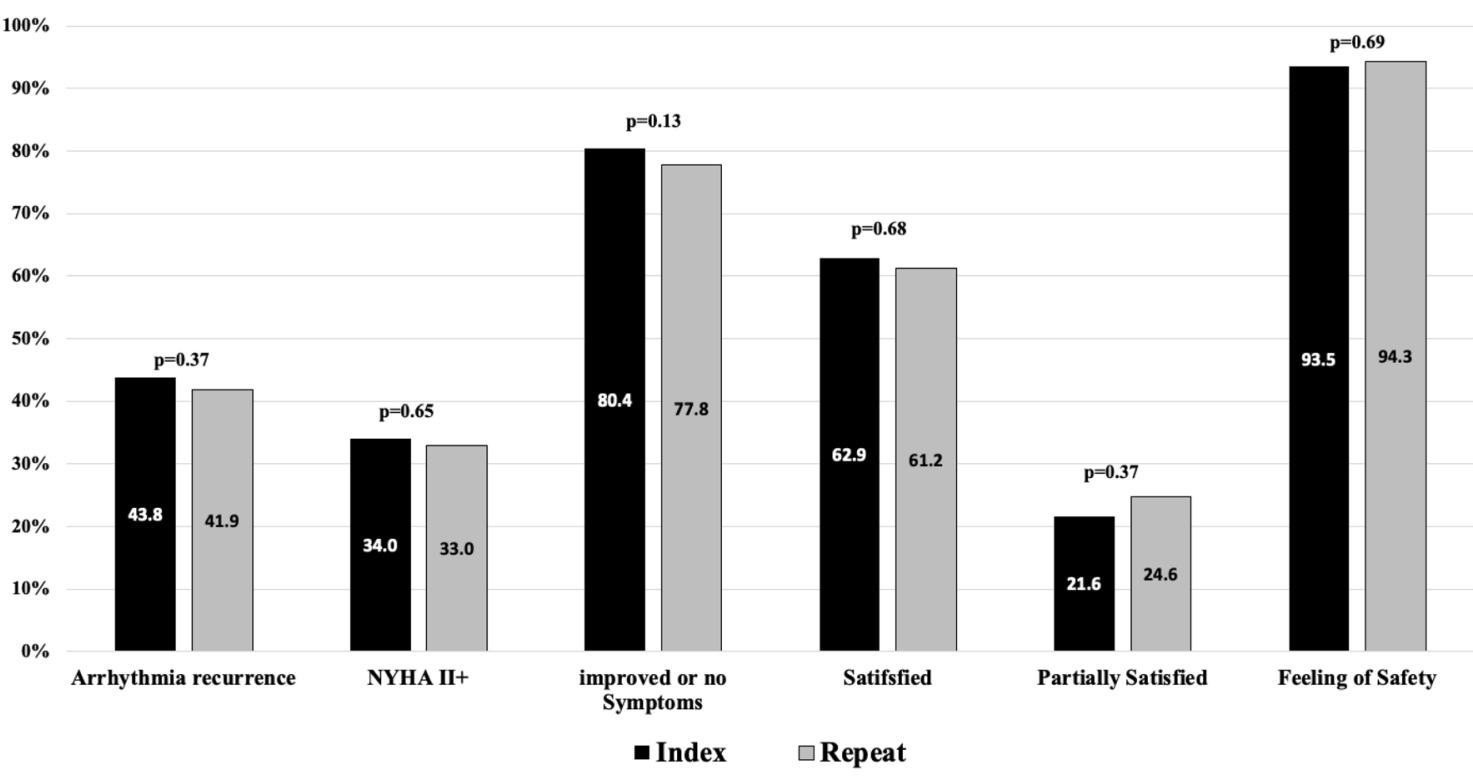

Fig. 2 Follow-up of Arrhythmia Recurrence, Symptoms, and Patient-oriented outcomes. NYHA: New York Heart Association. $P$ value $<0.05$ was considered significant

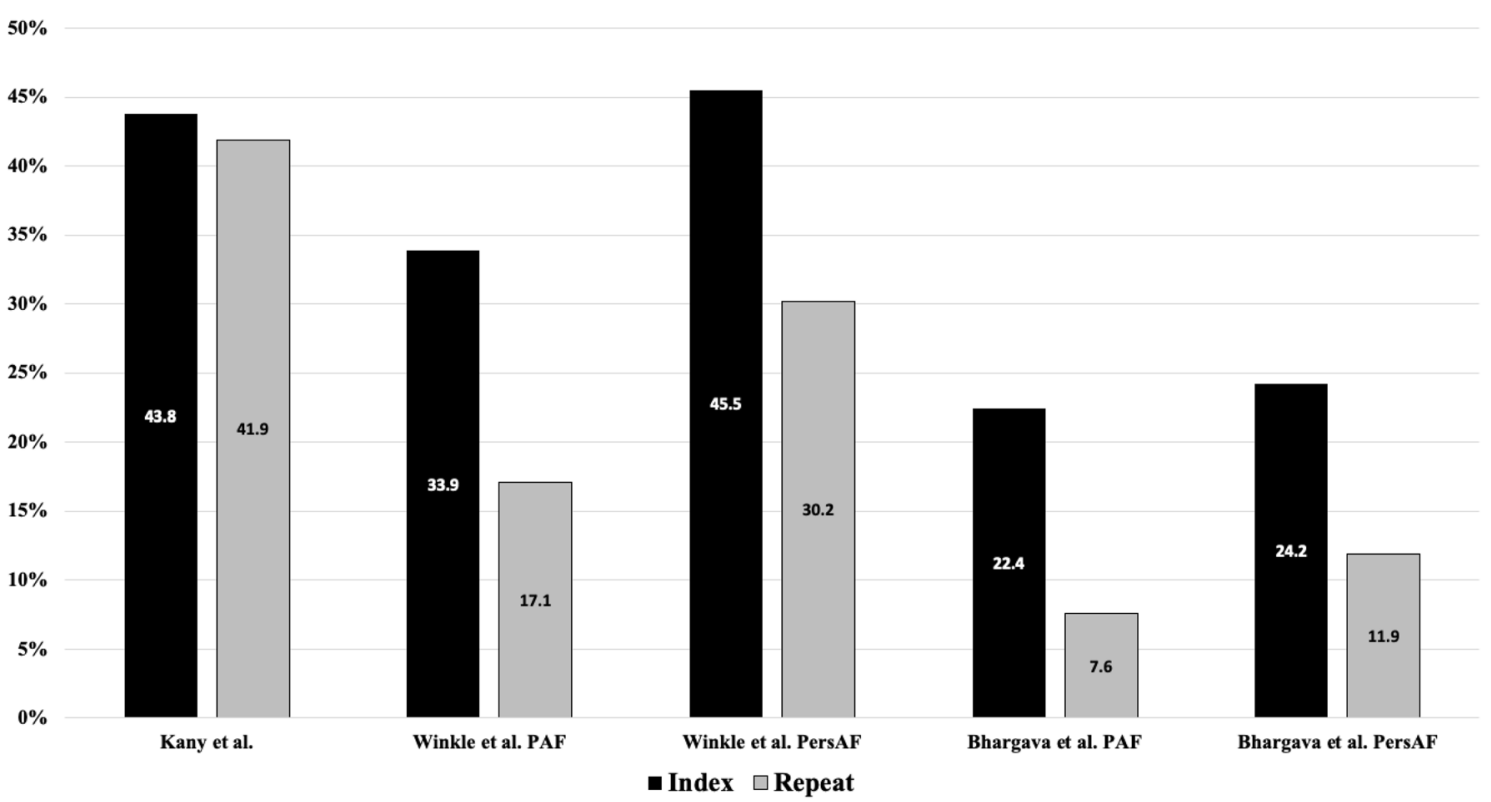

Fig. 3 Comparison of arrhythmia recurrence to other index vs repeat catheter ablation cohorts. Respective works (Winkle et al. [25] and Bhargava et al. [26]) showing arrhythmia recurrence for paroxysmal atrial fibrillation (PAF) and persistent atrial fibrillation (PersAF)

is more symptomatic than the collectives enrolled in large trials. In a sub-analysis of the CABANA trial, it was shown that AF ablation and anti-arrhythmic drug therapy are effective in reducing symptomatic burden with the former having significantly higher benefits as defined by the Atrial Fibrillation Effect on Quality of Life (AFEQT) Score (adjusted difference, 5.3 points [95\% CI 3.7-6.9]; $P<0.001$ ) [14]. There are, however, no data comparing the significant amount of repeat ablation to index procedures regarding procedurerelated outcomes. We show that patients report equally high rates of improved or no symptoms after index ablation as well as after repeat ablation ( $80.4 \%$ vs $77.8 \%)$. However, both groups reported significant incidence of $\geq$ NYHA II symptoms 1 year after index (34\%) or repeat (33\%) ablation. Compared to baseline, our data show that repeat ablation reduces the number of patients experiencing severe 
Table 4 Medical therapy at discharge

\begin{tabular}{|c|c|c|c|}
\hline Variable & Index $(n=3372)$ & Repeat $(n=735)$ & $p$ value \\
\hline \multicolumn{4}{|c|}{ Anti-arrhythmic drug (AAD) therapy } \\
\hline Beta-blocker & $74.5 \%$ & $70.2 \%$ & 0.017 \\
\hline AAD class I & $32.6 \%(1099 / 3372)$ & $30.1 \%(221 / 735)$ & 0.18 \\
\hline AAD class III & $21.4 \%(722 / 3372)$ & $24.4 \%(179 / 735)$ & 0.081 \\
\hline Sotalol & $18.3 \%(132 / 722)$ & $18.4 \%(33 / 179)$ & 0.96 \\
\hline Amiodarone & $79.2 \%(572 / 722)$ & $78.8 \%(141 / 179)$ & 0.89 \\
\hline $\begin{array}{c}\text { AAD class III } \\
\text { (added after } \\
\text { procedure) }\end{array}$ & 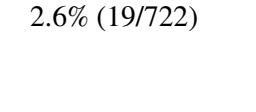 & $280^{\circ}$ & 0.90 \\
\hline AAD class IV & $1.6 \%(53 / 3372)$ & $3.4 \%(25 / 735)$ & $<0.001$ \\
\hline \multicolumn{4}{|l|}{$\begin{array}{l}\text { Antithrombotic } \\
\text { therapy }\end{array}$} \\
\hline ASA & $9.0 \%(304 / 3372)$ & $6.7 \%(49 / 735)$ & 0.040 \\
\hline Clopidogrel & $2.7 \%(91 / 3372)$ & $2.0 \%(15 / 735)$ & 0.31 \\
\hline $\begin{array}{l}\text { Vitamin } \mathrm{K} \\
\text { antagonist }\end{array}$ & $89.6 \%(3023 / 3373)$ & $91.0 \%(669 / 735)$ & 0.26 \\
\hline Heparin (UFH) & $8.3 \%(280 / 3372)$ & $3.9 \%(29 / 735)$ & $<0.001$ \\
\hline Heparin (LMWH) & $63.3 \%(2136 / 3372)$ & $70.3 \%(517 / 735)$ & $<0.001$ \\
\hline
\end{tabular}

$A S A$ acetylsalicylic acid; $U F H$ unfractionated heparin; $L M W H$ lowmolecular-weight heparin; All values given as percentages with total numbers in brackets. $P$ values were calculated by Fisher's exact test and considered significant if $<0.05 . *$

symptoms. Considering that current guidelines emphasize patient choice for treatment indications, patient satisfaction is an increasingly important procedure-related outcome [15]. Both patient cohorts undergoing index or repeat ablation report being either fully satisfied $(62.9 \%$ vs $61.2 \%)$ or partially satisfied ( $21.6 \%$ vs $24.7 \%)$. These data are comparable to other reported high incidences of patient satisfaction after AF ablation [7, 16]. Furthermore, less female patients in the repeat group suggest that women are less likely to be offered repeat ablation. An earlier analysis of the German Ablation Registry cohort showed that women were, in fact, more likely to have AF recurrence and more complications after catheter ablation [17].

PVI remains the only ablation strategy in AF with a proven effect on freedom of arrhythmia in long-term outcome. In our cohort, the repeat group was treated with additional lesions or CFAE significantly more often than the index group. However, the repeat group also had a higher number of patients with persistent AF $(30.7 \%$ vs $38.1 \%)$. Catheter ablation in persistent AF (PersAF) and long-standing persistent AF is significantly less successful in maintaining SR than in PAF [18]. The STAR-AF II trial showed no benefit of additional liner lesions or CFAE with PVI in PersAF [19]. The data studied in this investigation include procedures done before the publication of STARAF II and has therefore to be seen in that context. The FIRE and ICE Redo study reported that $15 \%$ of patients undergoing index ablation are undergoing repeat ablation within a single year, comparable to the $20 \%$ described before $[4,20]$. Ganesan et al. showed an average of 1.51 ablation procedures in a metanalysis of long-term outcome after ablation [18]. Therefore, safety in repeat procedures is of utmost importance. This study shows that in repeat ablation, despite additional lesions, EP study duration, fluoroscopy duration, and dose area product are significantly lower compared to index ablation. This finding is explained by the need for new circumferential wide area ablation at index ablation versus focused re-isolation in repeat cases. Consequently, less RF application time was noted in repeat ablation. This is in line with more recent data on repeat ablations from the CIRCA-DOSE trial [21]. Compared to other studies with similar comparisons, recurrence rates were higher in our study and generally in line with data from CIRCA-DOSE [3].

Furthermore, short-term complication or long-term MACE, MACCE, or composite safety were not different between repeat and index ablation. In line with existing data, we show that repeat ablation is safe in regard to procedural details, short- or long-term safety profile in a large, real-world cohort [6, 20, 22].

\section{Limitations}

The German Ablation Registry gives insights into a large, prospective real-world cohort undergoing catheter ablation. Yet, there are some limitations. First, the procedures were carried out between 2007 and 2010, and technical aspects have, at least in part, been improved in AF ablation [23]. Major advancements include contact force catheters, new EAM systems, new RF protocols like high-powershort-duration, or new generations of cryoballoon devices $[4,24]$. Another important difference is the change of periprocedural anticoagulation as uninterrupted regimes are favored currently compared with 2010 .

Second, in a registry-based cohort, important confounding factors have to be considered and may not be known. Patients undergoing index ablation are not necessarily the same patients in the repeat ablation group. Therefore, the characteristics of the repeat group might be influenced by a selection bias (patients with persistent $\mathrm{AF}$ are more likely to receive a repeat ablation). Follow-up was also only centralized at the 1 year by IHF and left to each participating center before that. This was usually done in clinical routine and therefore not standardized $(24 \mathrm{~h}$ vs $72 \mathrm{~h}$ Holter monitoring) and recurrence rates are therefore likely underestimated.

Complications and follow-up were also based on voluntary reports by health care workers and patients, a 
reporting bias has to be considered. Procedures and postprocedure care were dependent on each institute's local standards and are therefore heterogenous in nature.

\section{Conclusion}

Repeat ablation may offer symptomatic relief in patients with atrial fibrillation with high safety. Despite progressive forms of AF and more severe symptoms, the effects seem to be comparable to index ablation.

\section{Conflicts of interest}

SK: none; JS and MH: the long-term follow-up and a prior publication were partially supported by unrestricted grants from Medtronic, Biosense Webster, and Biotronik; LE: received research support from several drug and device companies active in the field of electrophysiolopgy and received honoraria from several such companies in the past; D.T.: receiving lecture fees/honoraria from Bayer Vital, Boehringer Ingelheim Pharma, Bristol-Myers Squibb, Daiichi Sankyo, Medtronic, Pfizer Pharma, Sanofi-Aventis, St. Jude Medical and ZOLL CMS; LT.: lecture fees/honoraria from Medtronic, Abbott, Boston Scientific, Biotronik; AR received travel grants and lecture fees from Biosense Webster, Medtronic, Ablamap, Böhringer Ingelheim, Cardiofocus, EPD/Philips.

\section{Ethical approval}

Ethics committee of the Rhineland-Palatinate State Medical Council (Landesärztekammer Rheinland-Pfalz) approved the study (No. 837.026.07 (5561)).

\section{Consent for publication}

Given by all co-authors.

Funding Open Access funding enabled and organized by Projekt DEAL. This work was funded by an unrestricted grant from foundation 'Stiftung Institut für Herzinfarktforschung Ludwigshafen' (Ludwigshafen, Germany).

Data availability All data and materials available at IFH Ludwigshafen.

Open Access This article is licensed under a Creative Commons Attribution 4.0 International License, which permits use, sharing, adaptation, distribution and reproduction in any medium or format, as long as you give appropriate credit to the original author(s) and the source, provide a link to the Creative Commons licence, and indicate if changes were made. The images or other third party material in this article are included in the article's Creative Commons licence, unless indicated otherwise in a credit line to the material. If material is not included in the article's Creative Commons licence and your intended use is not permitted by statutory regulation or exceeds the permitted use, you will need to obtain permission directly from the copyright holder. To view a copy of this licence, visit http://creativecommons.org/licenses/by/4.0/.

\section{References}

1. Schnabel RB, Yin $X$, Gona $P$ et al (2015) 50 year trends in atrial fibrillation prevalence, incidence, risk factors, and mortality in the Framingham Heart Study: a cohort study. Lancet 386(9989):154162. https://doi.org/10.1016/S0140-6736(14)61774-8

2. Hindricks G, Potpara T, Dagres N et al (2020) 2020 ESC guidelines for the diagnosis and management of atrial fibrillation developed in collaboration with the European Association of CardioThoracic Surgery (EACTS): the task force for the diagnosis and management of atrial fibrillation of the European Society of Cardiology (ESC) developed with the special contribution of the European Heart Rhythm Association (EHRA) of the ESC. Eur Heart J. https://doi.org/10.1093/eurheartj/ehaa612

3. Andrade JG, Champagne J, Dubuc M et al (2019) Cryoballoon or radiofrequency ablation for atrial fibrillation assessed by continuous monitoring: a randomized clinical trial. Circulation 140(22):1779-1788. https://doi.org/10.1161/CIRCULATIO NAHA.119.042622

4. Kuck K-H, Brugada J, Fürnkranz A et al (2016) Cryoballoon or radiofrequency ablation for paroxysmal atrial fibrillation. $\mathrm{N}$ Engl J Med 374(23):2235-2245. https://doi.org/10.1056/NEJMoa1602 014

5. Rottner L, Bellmann B, Lin T et al (2020) Catheter ablation of atrial fibrillation: state of the art and future perspectives. Cardiol Ther. https://doi.org/10.1007/s40119-019-00158-2 ((Published online January 2, 2020))

6. Chun KRJ, Schmidt B, Kuck K-H et al (2013) Catheter ablation of atrial fibrillation in the young: insights from the German Ablation Registry. Clin Res Cardiol 102(6):459-468. https://doi. org/10.1007/s00392-013-0553-6

7. Fink T, Metzner A, Willems S et al (2019) Procedural success, safety and patients satisfaction after second ablation of atrial fibrillation in the elderly: results from the German Ablation Registry. Clin Res Cardiol 108(12):1354-1363. https://doi.org/10.1007/ s00392-019-01471-5

8. Eitel C, Ince H, Brachmann J et al (2019) Atrial fibrillation ablation strategies and outcome in patients with heart failure: insights from the German ablation registry. Clin Res Cardiol 108(7):815823. https://doi.org/10.1007/s00392-019-01411-3

9. Wasmer K, Hochadel M, Wieneke H et al (2019) Long-term symptom improvement and patient satisfaction after AV-node ablation vs. pulmonary vein isolation for symptomatic atrial fibrillation: results from the German Ablation Registry. Clin Res Cardiol. 108(4):395-401. https://doi.org/10.1007/s00392-018-1368-2

10. Anker SD, Agewall S, Borggrefe M et al (2014) The importance of patient-reported outcomes: a call for their comprehensive integration in cardiovascular clinical trials. Eur Heart J 35(30):20012009. https://doi.org/10.1093/eurheartj/ehu205

11. Van Gelder IC, Hagens VE, Bosker HA et al (2002) A comparison of rate control and rhythm control in patients with recurrent persistent atrial fibrillation. N Engl J Med 347(23):1834-1840. https://doi.org/10.1056/NEJMoa021375

12. Singh BN, Singh SN, Reda DJ et al (2005) Amiodarone versus sotalol for atrial fibrillation. N Engl J Med 352(18):1861-1872. https://doi.org/10.1056/NEJMoa041705 
13. Packer DL, Mark DB, Robb RA et al (2019) Effect of catheter ablation vs antiarrhythmic drug therapy on mortality, stroke, bleeding, and cardiac arrest among patients with atrial fibrillation: the CABANA randomized clinical trial. JAMA 321(13):1261. https://doi.org/10.1001/jama.2019.0693

14. Mark DB, Anstrom KJ, Sheng S et al (2019) Effect of catheter ablation vs medical therapy on quality of life among patients with atrial fibrillation: the CABANA randomized clinical trial. JAMA 321(13):1275. https://doi.org/10.1001/jama.2019.0692

15. Kirchhof P, Benussi S, Kotecha D et al (2016) 2016 ESC Guidelines for the management of atrial fibrillation developed in collaboration with EACTS. Eur Heart J 37(38):2893-2962. https:// doi.org/10.1093/eurheartj/ehw210

16. Ikemura N, Kohsaka S, Kimura T et al (2019) Assessment of sex differences in the initial symptom burden, applied treatment strategy, and quality of life in Japanese patients with atrial fibrillation. JAMA Netw Open 2(3):e191145. https://doi.org/10.1001/jaman etworkopen.2019.1145

17. Zylla MM, Brachmann J, Lewalter T et al (2016) Sex-related outcome of atrial fibrillation ablation: insights from the German Ablation Registry. Heart Rhythm 13(9):1837-1844. https://doi. org/10.1016/j.hrthm.2016.06.005

18. Ganesan AN, Shipp NJ, Brooks AG et al (2013) Long-term outcomes of catheter ablation of atrial fibrillation: a systematic review and meta-analysis. J Am Heart Assoc. https://doi. org/10.1161/JAHA.112.004549

19. Verma A, Jiang C, Betts TR et al (2015) Approaches to catheter ablation for persistent atrial fibrillation. N Engl J Med 372(19):1812-1822. https://doi.org/10.1056/NEJMoa1408288
20. Kuck K-H, Albenque J-P, Chun KRJ et al (2019) Repeat ablation for atrial fibrillation recurrence post cryoballoon or radiofrequency ablation in the FIRE AND ICE trial. Circ Arrhythm Electrophysiol. https://doi.org/10.1161/CIRCEP.119.007247

21. Cheung CC, Deyell MW, Macle L et al (2020) Repeat atrial fibrillation ablation procedures in the CIRCA-DOSE Study. Circ Arrhythm Electrophysiol. https://doi.org/10.1161/CIRCE P.120.008480

22. Ammar-Busch S, Kaess BM, Bruhm A et al (2015) Atrial tachycardias following persistent atrial fibrillation ablation: predictors of recurrence after the repeat ablation: atrial tachycardias following persistent atrial fibrillation ablation. J Cardiovasc Electrophysiol 26(12):1315-1320. https://doi.org/10.1111/jce.12817

23. Santoro F, Metzner A, Brunetti ND et al (2019) Left atrial anterior line ablation using ablation index and inter-lesion distance measurement. Clin Res Cardiol 108(9):1009-1016. https://doi. org/10.1007/s00392-019-01428-8

24. Kottkamp H, Bender R, Berg J (2015) Catheter ablation of atrial fibrillation. J Am Coll Cardiol 65(2):196-206. https://doi. org/10.1016/j.jacc.2014.10.034

25. Winkle RA, Mead RH, Engel G, Patrawala RA (2011) Long-term results of atrial fibrillation ablation: the importance of all initial ablation failures undergoing a repeat ablation. Am Heart $\mathbf{J}$ 162(1):193-200. https://doi.org/10.1016/j.ahj.2011.04.013

26. Bhargava M, Di Biase L, Mohanty P et al (2009) Impact of type of atrial fibrillation and repeat catheter ablation on long-term freedom from atrial fibrillation: results from a multicenter study. Heart Rhythm 6(10):1403-1412. https://doi.org/10.1016/j.hrthm .2009 .06 .014 